\title{
Virtual Fixture Control of a Hybrid Parallel-Serial Robot for Assisting Ophthalmic Surgery: an Experimental Study
}

\author{
M. A. Nasseri ${ }^{1}$, P. Gschirr ${ }^{1}$, M. Eder ${ }^{1}$, S. Nair ${ }^{1}$, K. Kobuch ${ }^{2}$, M. Maier ${ }^{2}$, D. Zapp ${ }^{2}$, C. Lohmann ${ }^{3}$ and A. Knoll ${ }^{2}$
}

\begin{abstract}
This paper presents the virtual fixture control methods for a hybrid parallel-serial micromanipulator, which is designed for assisting ophthalmic surgeons. Virtual fixtures are features of surgical robotic setups to improve quality of the surgery and reduces the operation risk. In the domain of ophthalmic surgery lack of virtual fixtures in manual operations has limited, and sometimes even blocked, the treatment options. The contribution of this paper is concept analysis and implementation of flexible virtual fixture for the novel hybrid parallel-serial mechanism and experimentally evaluation of this concept. The virtual fixture using this mechanism enables the user to adjust them even during the procedure. Pivoting around a Remote Center of Motion (RCM), which in retinal surgery is the incision point, is the most famous virtual fixture of ophthalmic surgery. Autonomous RCM adjustment for VitreoRetinal surgery, implying retinal reachability study, is the secondary contribution which is investigated in this paper.
\end{abstract}

\section{INTRODUCTION}

Since the 1970s when the first pars plana vitrectomy was performed [1], there has been an important change in trends in ophthalmic operations, particularly in VitreoRetinal surgery. Not only have the outcomes of these surgeries been improved, but nowadays, it is also possible to find cure to ocular conditions that were untreatable before. The increasing positive results in the ophthalmic surgery are mostly due to new and better developed surgical techniques, improved low-gauge instrumentation, high-speed cutters and upgraded and enhanced visualization tools. Nevertheless, the success of these procedures is still limited by the surgeons' precision and dexterity. In this line, it is the employment of assisting robots what sets a break through the barrier of human abilities. The abilities barrier which is discussed in this paper is virtual fixture control. To maximize the quality and safety of operations the virtual fixtures should be realized. For instance Remote Center of Motion (RCM) guarantees that during the Vitreo-Retinal surgery there won't be a damage to sclera. Or the collision avoidance of lens and retina helps the surgeons to avoid unwanted touching of sensitive regions (See Fig.1). A clinically acceptable

\footnotetext{
*This work was supported by TUM Graduate School of Information Science in Health

${ }^{1}$ M. Ali Nasseri, P. Gschirr, S. Nair, M. Eder and A. Knoll are with the Department of Robotics and Embedded Systems, Institut für Informatik, Technische Universität München nasseri, gschirr, nair, ederma, knoll at in.tum.de

${ }^{2}$ K. Kobuch, M. Maier, D. Zapp and C. P. Lohmann are with the Augenklinik rechts der Isar, Technische Universität München karin.kobuch, mathias.maier, daniel.zapp, c.lohmann at mri.tum.de
}

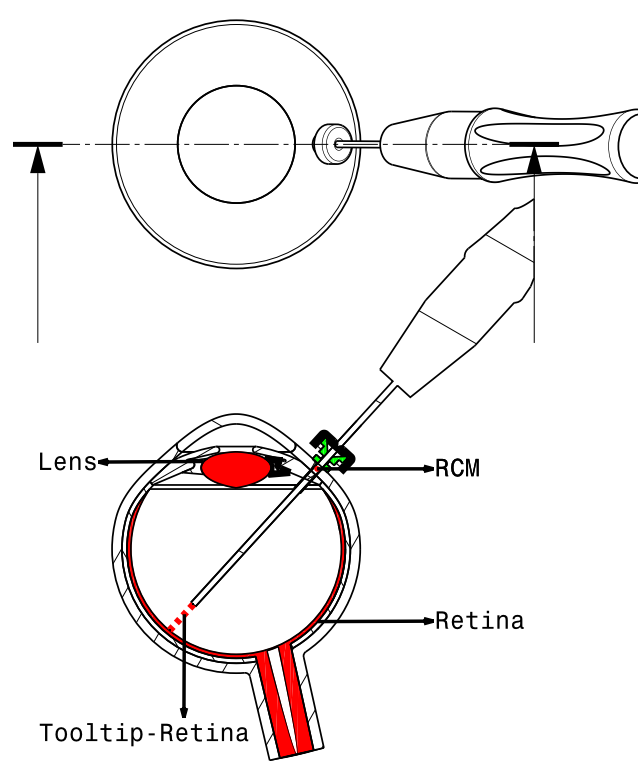

Fig. 1. Ophthalmic tool introduced into the eye ball using micro cannula (Vitreo-Retinal surgery). The most famous virtual fixtures are marked

surgical robot should be able to satisfy virtual fixtures. This experimental study investigates the virtual fixture capabilities of the ophthalmic micromanipulator from Technische Universität München. This tiny robot which is introduced in [2] has a hybrid parallel-serial configuration and is specifically designed for Vitreo-Retinal surgery.

Related Works: Virtual fixture is a common problem in Minimally Invasive Surgery(MIS). There are three methods to solve virtual fixture problems in the robotics literature; 1- Mechanically constrained virtual fixtures (see e.g. [3]): In this method the kinematics of the robot is designed in a way that physically limits the motions. This method, due to hardware constraints, is considered to have the highest reliability and safety. However, it has no flexibility and consequently is not intuitive. 2- Semi Mechanically constrained virtual fixtures (see e.g. [4]): In this method the kinematics of the robot provides dependencies which together with controller satisfies virtual fixture constraints. This method is more flexible than the first one but there still are degrees of freedom reductions which limits the free motion. 3- Control based virtual fixtures (see e.g. [5]): Normally in this method the robot's kinematics is capable of 6DOF motion. The control algorithms are used to define virtual fixtures when it is needed. It provides the maximum flexibility but the reliability of the controller should be carefully verified for 


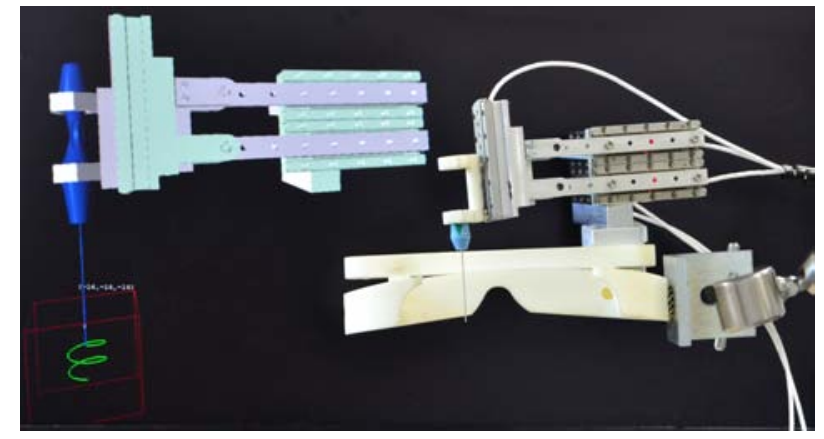

Fig. 2. TUM Ophthalmic Micromanipulator (right) and Simulator (left): Serial configuration of parallel joints

safety reasons.

Flexibility of the virtual fixtures plays an important role for Vitreo-Retinal surgery. During the operation the surgeon needs to change the virtual fixtures. For instance for the needle approach and insertion phase the surgeon needs at least five degrees of freedom to locate the needle. When the needle is inserted into the microcannula the RCM needs to be set at the incision point. The RCM position needs to be changed several times to keep the line of sight of the microscope and meanwhile access the points of interest on the surface of the retina. The surgeon should also be able to enable/disable other virtual fixtures (Tool-retina distance, Lens collision avoidance, ...). The contribution of this paper is investigating the capability of the robot from [2] for realizing controlled based virtual fixtures. The investigation includes the mathematical modeling as well as the experimental evaluation. Furthermore, the reachability analysis result, which is discussed in this paper, is used as a reference for automatic adjustment of the RCM.

Organization of this Paper: The remainder of this paper is structured as follows: In section 2 the models of the Eye and the Robot are explained. The methods for solving virtual fixture problems is described in section 3. The implemented methods are discussed in section 4 which is followed by results in section 5. Section 6 describes the experimental evaluation and clinical experiments and this paper is going to be concluded in section 7 .

\section{MODELS}

\section{A. Model of the Robot}

Fig.2 shows the robot which is used in this study. It consists of a novel serial configuration of parallel coupled joint mechanisms. The detailed kinematics and mechanical models of the robot was analyzed in [2], in this paper the feasibility of the adjustable RCM is discussed. Five sub micron precision piezo actuators are used to drive the robot and it is controlled using a middle-ware based architecture [6].

\section{B. Model of the Eye}

The Eye ball in this work is modeled as a simple sphere with a diameter of $24.2 \mathrm{~mm}$ [13]. The cannula for inserting the tool into the eye is placed about $3.5 \mathrm{~mm}$ away from the cornea [9] and defines the location of the RCM. Additionally, another constraint is taken into account: because the surgeon uses the microscope during intra-ocular operations, it is essential that the areas which are accessible overlap with the field of view of the microscope. For this it is assumed that the inner eye is visible through a circular hole defined by the pupil without refractions of the lens. The radius of the circular hole is set to the average radius of the cornea. Fig. 3 shows the relevant parameters of the model. The diameter of the eyeball and the cornea are taken from [13].

By tilting the eye about its center the RCM is relocated and the line of sight transforms accordingly as displayed in Fig. 5. In our analysis we assume a maximum rotation of the eye $\rightarrow 30$ degrees about the $z$-axis, maximum rotation in the direction of the cannula (around the $x$-axis) $\rightarrow-15$ degrees and the maximum rotation around the $y$-axis $\rightarrow \pm 10$ degrees.

\section{Methodology}

The restriction of motions through the entry point of the patient's body is one of the most important characteristics of minimally invasive surgery assisted by robots. More specifically, the link penetrating the tissue is only allowed to translate along its axis and rotate about the entry point. This reduces stress on the tissue and thus accelerates the healing process after the surgery. For ophthalmic-VitreoRetinal surgery the RCM is especially useful to maintain the eyeball in a certain position to perform operations on the retina. As the surgeon uses the microscope to look at the retina through the widened pupil the position of the eye should be maintained while moving the tooltip. This is only possible by restricting the motion of the last link with respect to the entry point on the sclera. We follow the general approach in [7] and adapt it to our setting. One major advantage of this approach in comparison to [8] is that it does not require the definition of a tangent plane at the entry point.

For this setup the RCM is, but not necessarily, located on the axis of the tool shaft (See Fig.1) and its position can be written as:

$$
\mathbf{p}_{\text {rcm }}=\mathbf{p}_{5}+\lambda\left(\mathbf{p}_{\text {tool }}-\mathbf{p}_{5}\right)
$$

Deriving the equation with respect to time yields:

$$
\dot{\mathbf{p}}_{r c m}=\dot{\mathbf{p}}_{5}+\lambda\left(\dot{\mathbf{p}}_{\text {tool }}-\dot{\mathbf{p}}_{5}\right)+\dot{\lambda}\left(\mathbf{p}_{\text {tool }}-\mathbf{p}_{5}\right)
$$

Given the Jacobians $\mathbf{J}_{r \mathrm{rm}}$ and $\mathbf{J}_{5}$ at the respective points $\mathbf{p}_{\mathrm{rcm}}$ and $\mathbf{p}_{5}$, the equation can be reformulated by making use of
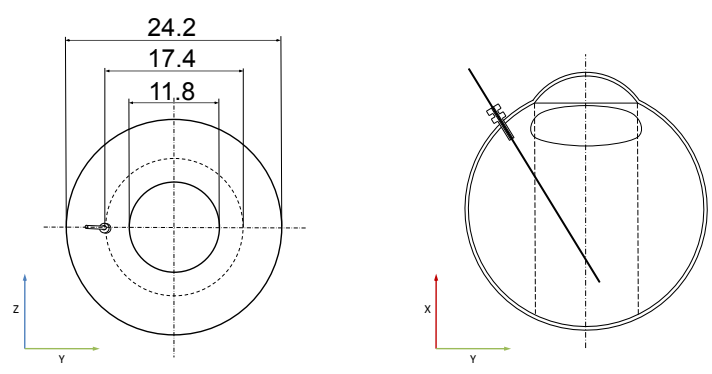

Fig. 3. The eye model used in our analysis. 


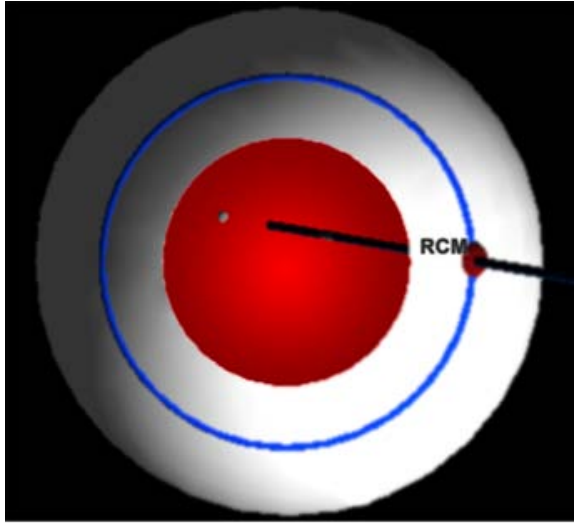

Fig. 4. Evaluation run in the simulation environment. The grey dot indicates the point on the retina the tool is aimed at.
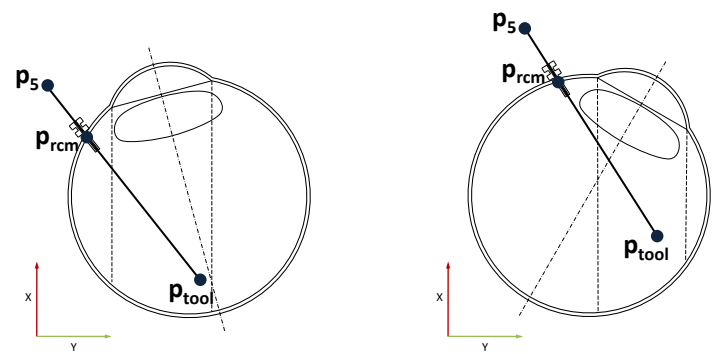

Fig. 5. Different rotations of the eye, Left: $-10^{\circ}$ about $z$-axis, Right: $-30^{\circ}$ about $z$-axis.

the fact that $\dot{\mathbf{p}}_{i}=\mathbf{J}_{i} \dot{\mathbf{q}}$ :

$$
\dot{\mathbf{p}}_{r c m}=\mathbf{J}_{5} \dot{\mathbf{q}}+\lambda\left(\mathbf{J}_{t o o l} \dot{\mathbf{q}}-\mathbf{J}_{5} \dot{\mathbf{q}}\right)+\dot{\lambda}\left(\mathbf{p}_{\text {tool }}-\mathbf{p}_{5}\right)
$$

In matrix form this can be written as

$$
\dot{\mathbf{p}}_{r c m}=\left(\begin{array}{c}
\mathbf{J}_{5}+\lambda\left(\mathbf{J}_{t o o l}-\mathbf{J}_{5}\right) \\
\mathbf{p}_{\text {tool }}-\mathbf{p}_{5}
\end{array}\right)^{T}\left(\begin{array}{l}
\dot{\mathbf{q}} \\
\dot{\lambda}
\end{array}\right)=\mathbf{J}_{r c m}\left(\begin{array}{l}
\dot{\mathbf{q}} \\
\dot{\lambda}
\end{array}\right)
$$

With this formulation it is possible to restrict the motion of the RCM by forcing its velocity to zero i.e.

$$
\dot{\mathbf{p}}_{r c m}=\mathbf{J}_{r c m}\left(\begin{array}{l}
\dot{\mathbf{q}} \\
\dot{\lambda}
\end{array}\right) \stackrel{!}{=} \mathbf{0}_{3 \times 1}
$$

with $\mathbf{J}_{r c m} \in \mathbb{R}^{3 \times 6}$. By fulfilling the RCM constraint the degrees of freedom of the robot are reduced by two, i.e. for fulfilling a task in an $n_{t}$-dimensional space the robot must have at least $n \geq n_{t}+2$ degrees of freedom [7].

Control Design: To satisfy the RCM constraint while moving the tooltip within the eye:

$$
\dot{\mathbf{q}}=\mathbf{J}^{\dagger} \mathbf{K e}
$$

For incorporating the RCM constraint two methods are usually used. The first is based on the concept of taskpriority [10] as implemented in [8] and the second on the alternative kinematics [11] (also referred to as extended Jacobian footnote distinguishing [12]) approach as shown in [7] [14].

In this work the second approach was followed; using the alternative kinematics where the RCM constraint can be directly incorporated into the robot task. The robot has five degrees of freedom from which two are used to fulfill the RCM constraint. As a result there are three degrees of freedom left, which match the three-dimensional workspace necessary to place the tooltip within the eyeball.

The robot task with coordinates $\mathbf{x}_{t}=\left(\begin{array}{lll}x & \phi & \psi\end{array}\right)^{T}$ is expressed. The task space is defined by $\mathscr{T}=\mathbb{R} \times S^{2}$. Hence the robot task is defined as the Cartesian $x$ position and the two angles $\phi$ and $\psi$. The joint velocities are related to the task velocities through:

$$
\dot{\mathbf{x}}_{t}=\mathbf{J}_{t} \dot{\mathbf{q}}
$$

where $\mathbf{J}_{t} \in \mathbb{R}^{3 \times 6}$ is the Jacobian derived from the kinematic mapping. Taking the RCM constraint into account the extended task can be defined as:

$$
\begin{aligned}
\mathbf{x}_{\text {ext }} & =\left(\begin{array}{lllll}
\mathbf{x}_{t}^{T} & \mathbf{p}_{r c m}^{T}
\end{array}\right)^{T} \\
& =\left(\begin{array}{llllll}
x & \phi & \psi & x_{r c m} & y_{r c m} & z_{r c m}
\end{array}\right)^{T} \in \mathbb{R} \times S^{2} \times \mathbb{R}^{3}
\end{aligned}
$$

And the kinematics of the extended task are then given by:

$$
\dot{\mathbf{x}}_{e x t}=\left(\begin{array}{cc}
\mathbf{J}_{t} & \mathbf{0}_{3 \times 1} \\
& \mathbf{J}_{r c m}
\end{array}\right)\left(\begin{array}{l}
\dot{\mathbf{q}} \\
\dot{\lambda}
\end{array}\right)=\mathbf{J}_{e x t}\left(\begin{array}{c}
\dot{\mathbf{q}} \\
\dot{\lambda}
\end{array}\right)
$$

Assuming a desired robot task $\mathbf{x}_{d}$ and the position of the $\mathrm{RCM}$, which is defined by the location of the trocar $\mathbf{p}_{\text {trocar }}$ the extended task error is given by:

$$
\mathbf{e}_{\text {ext }}=\left(\begin{array}{c}
\mathbf{x}_{\text {ext }}-\mathbf{x}_{d} \\
\mathbf{p}_{\text {trocar }}-\mathbf{p}_{\text {rcm }}
\end{array}\right)
$$

Similar to the unrestricted movement the kinematic control is written as:

$$
\left(\begin{array}{c}
\dot{\mathbf{q}} \\
\dot{\lambda}
\end{array}\right)=\mathbf{J}_{\text {ext }}^{\dagger}\left(\begin{array}{cc}
\mathbf{K}_{t} & \mathbf{0}_{3 \times 3} \\
\mathbf{0}_{3 \times 3} & \mathbf{K}_{r c m}
\end{array}\right) \mathbf{e}_{e x t}
$$

where $\mathbf{K}_{t}$ and $\mathbf{K}_{r c m}$ are both postive definite diagonal matrices in $\mathbb{R}^{3 \times 3}$. The control law guarantees decoupled exponential convergence of the task to the desired value [7].

Algorithmic Singularities In the case of unconstrained movement it was already shown in [2] that the robot does not have any singularities within its workspace. However, with the augmented kinematics applied in the control design further singularities can be introduced due to rank deficiency or linear dependencies in the sub matrix $\mathbf{J}_{R C M}$ [12]. These are called algorithmic singularities and can be avoided by choosing the kinematic functions. In our control design we chose the angles $\psi$ and $\phi$ instead of $y$ and $z$ coordinates to avoid algorithmic singularities within the robot's workspace.

\section{DISCUSSION}

Reachability analysis: For the robot to be used in practice it is essential that its workspace covers the regions of the eye that have to be accessible during a surgical procedure.

Considering only translational movement, that is parallel movement of the first four piezo positioners with travel ranges of $\pm 15 \mathrm{~mm}$, the workspace is limited by a cube 
of dimensions $30 \times 30 \times 30 \mathrm{~mm}$. By assuming an average diameter of the eye of $24.2 \mathrm{~mm}$ this seems to be sufficient.

Effect of the RCM constraint: It is desirable that most
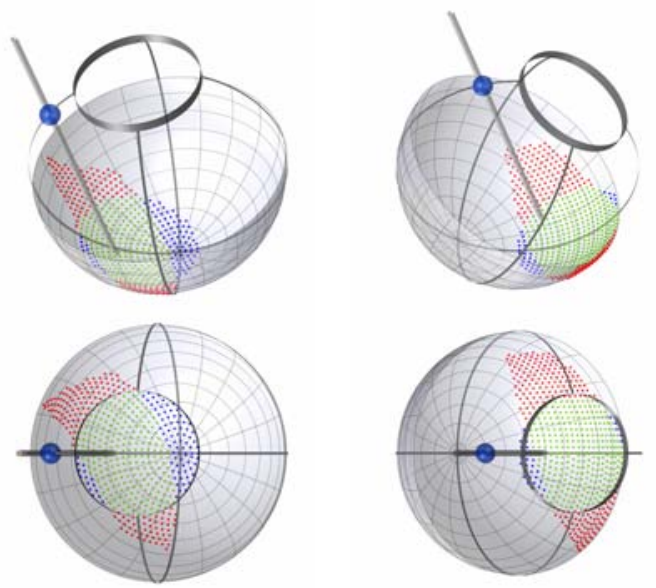

Fig. 6. Reachability Analysis, Blue, Red and green dots are showing Visible, Accessible and Visible-Accessible points respectively

of the points within the workspace are also accessible when enforcing an RCM constraint. However, enforcing an RCM constraint limits the workspace considerably depending on where it is placed. This is obviously due to the fact that two degrees of freedom are lost. Fig. 6 shows the effect of enforcing the RCM constraint at different locations. As a result, in order to access different regions of the retina the RCM location needs to be changed during a surgery accordingly. Another property that is connected to this issue is that in which configurations more visible-accessible points can be reached.

Reachability within the eye: To further assess the practicality of the robotic setup the reachability within the eye is investigated under different RCM constraints. More specifically the location of the eye with respect to the robot base is investigated, which then automatically defines the location of the RCM on the eye. Moreover, we also include the tilt of the robot about the $z$-axis. In our analysis we assume that by rotating the eye about its center the RCM location can be automatically determined.

Best location of the eye with respect to the robot base: For determining the best location of the eye with respect to the robot base as well as the necessary tilt of the robot we apply the following method:

- First, the location is determined experimentally until it yields satisfactory results within a certain predefined range of rotations of the eye.

- Second, the workspace of the robot is sampled. The sampled points are taken as an input for possible RCM locations and compared to the previously experimentally determined location.

In doing so the eye model is rotated by -10 to 30 degrees about the $z$-axis and -10 to 10 degrees about the $y$-axis. Intuitively the best flexibility of the robot is within the central $30 \times 30 \times 30 \mathrm{~mm}$ cube and thus an offset of the eye that covers

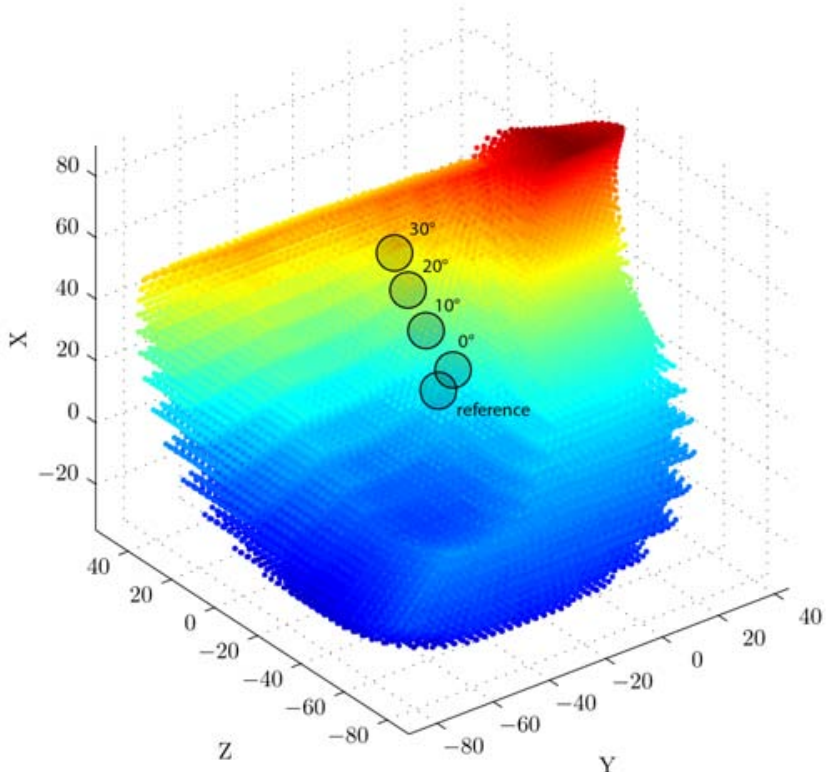

Fig. 7. Locations with the best performance for every tilt within the workspace. The marked angles are the tilt of the robot base with respect to the eye

this part of the workspace should yield good results. More specifically, the center of the eye is set to the location of the tooltip of the robot where all its positioners are in zero position. From this the RCM location is calculated and the analysis of reachable as well as visible areas within the eye is conducted. Fig. 6 shows the result of the analysis. The red dots represent areas that can be reached by the tool tip of the robot. Blue dots are those which are visible through the simplified circular hole. Green dots would indicate areas that are visible and reachable.

Comparison to other RCM candidates: To compare the reference with other candidates we sample the robot's workspace by positioning the five actuators in $3 \mathrm{~mm}$ steps in their working range of $\pm 15 \mathrm{~mm}$ and calculate the position of the end effector via forward kinematics. This yields $11^{5}=$ 161051 points. To get rid of configurations that yield much worse results than our reference we first filter the workspace by analysing the reachability of the robot without rotating the eye but by tilting the robot about the $z$-axis within the range of $0-30$ degrees with 10 degree steps. The filtered candidates are then more closely analysed within the full rotation range of the eye.

\section{REsults}

The candidates obtained for each tilt with the best overlap of visible and reachable areas, which we will refer to as coverage or performance, are chosen as the center of a sphere with diameter of $10 \mathrm{~mm}$. Candidates within this neighbourhood are considered by our analysis and are illustrated in Fig. 7 within the workspace. The exact locations of the candidates with the best performance are listed in Table I. Fig. 8 illustrates the average coverage and the standard deviation for each configuration within the region of interest. The results are summarized in Table II. All values correspond 
TABLE I

POINTS WITH THE BEST PERFORMANCE IN EACH TILT CONFIGURATION.

\begin{tabular}{|l|l|l|l|l|l|}
\hline & ref & $0^{\circ}$ & $10^{\circ}$ & $20^{\circ}$ & $30^{\circ}$ \\
\hline$x$ & -54.19 & -22.19 & -31.15 & -39.84 & -46.96 \\
\hline$y$ & 82.50 & 104.68 & 98.62 & 90.03 & 78.66 \\
\hline$z$ & 54.50 & 55.90 & 55.83 & 55.90 & 56.10 \\
\hline
\end{tabular}

to the percentage of overlap of visible and reachable points. As the results show, the best coverage of our reference is similar to those obtained by the analysis. However, the deviation is also very high and at worst only a coverage of around $45 \%$ is obtained. i.e. the region around the reference is very volatile. In a practical setting were the location of the eye cannot be positioned exactly at the desired coordinates this is crucial. Thus the regions of interest obtained in the analysis should be considered as where to roughly place the eye.

TABLE II

QUANTITATIVE RESULTS OF THE ANALYSIS.

\begin{tabular}{|l|l|l|l|l|l|}
\hline & ref & $0^{\circ}$ & $10^{\circ}$ & $20^{\circ}$ & $30^{\circ}$ \\
\hline Mean & 0.65 & 0.88 & 0.91 & 0.90 & 0.80 \\
\hline Deviation & 0.11 & 0.03 & 0.02 & 0.03 & 0.03 \\
\hline Max & 0.89 & 0.84 & 0.92 & 0.95 & 0.95 \\
\hline Min & 0.42 & 0.70 & 0.80 & 0.84 & 0.84 \\
\hline
\end{tabular}

\section{EXPERIMENTAL EVALUATION}

The elaborated model and its results are evaluated experimentally using the simulation environment and the area that can be reached experimentally is examined. The RCM is set manually, which inhibits to place it exactly at the position where the maximum coverage could be obtained. After fixing the RCM the needle is inserted into the eye and moved into all directions along the retina as far as possible. The positions are recorded and it is indicated whether the retina can be touched with the current orientation of the robot. One evaluation run for the best position without tilting the robot and no rotation of the eye is shown in Figure 9. The red area indicates the positions on the retina that can be reached.

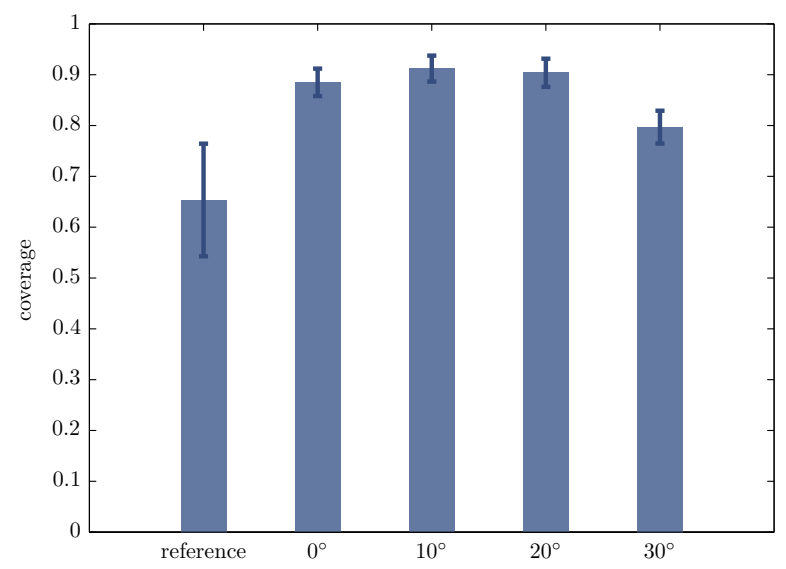

Fig. 8. Average performance of candidates and their standard deviation.

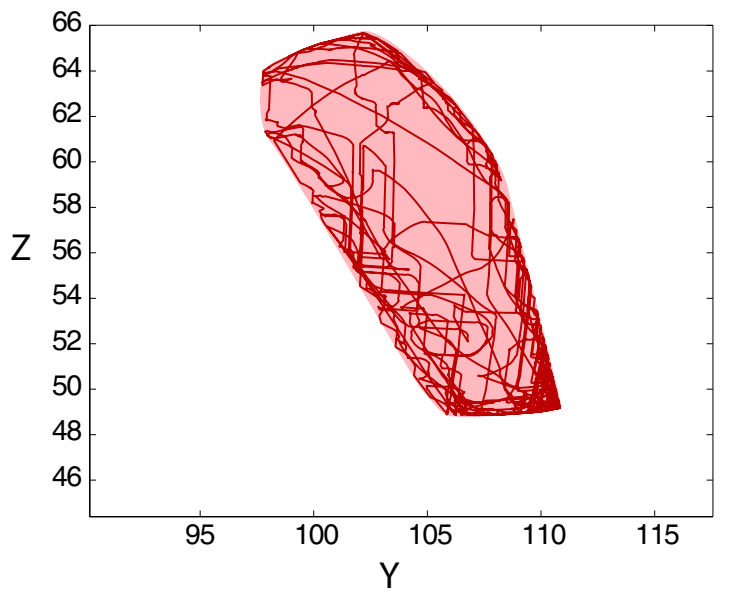

Fig. 9. Recorded trajectory of reachable points on the retina from one evaluation run. The shaded region indicates the reachable area derived from the trajectory.

This procedure is repeated for all configurations listed above, including rotations of the eye about the $z$-axis with $-10,0$ and 30 degrees. Then the area which was reached during the experiment is compared to the prediction from the model as presented in the former section. For simplicity, the areas are projected onto a plane.

The following indicators are used to quantitatively asses the results:

- Overlap of reachable areas $r$ :

$$
o_{r}=\frac{M_{r} \cap E_{r}}{M_{r} \cup E_{r}}
$$

where $M_{r}$ defines the reachable area predicted by the model $M$ and $E_{r}$ the area reached during the evaluation run $E$.

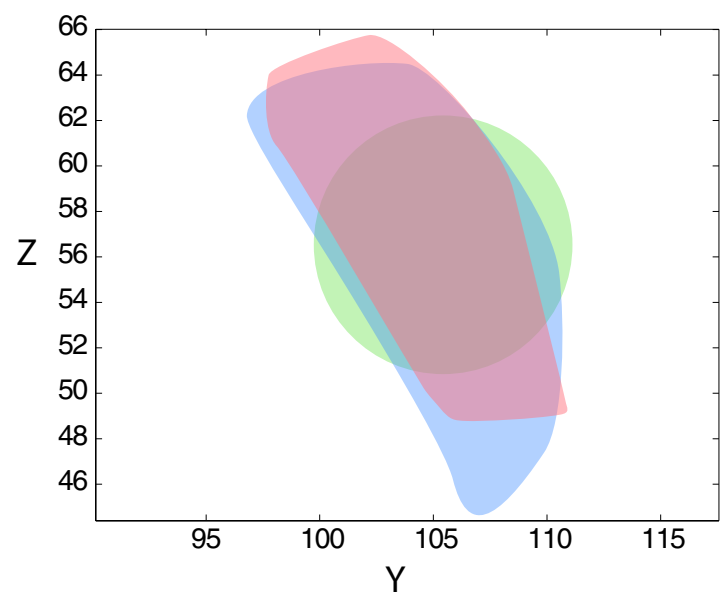

Fig. 10. The visible and reachable areas predicted by the model and determined during the evaluation run for the best location of the eye without tilting the robot(Green: Visible area, Blue: Reachable area from model and Red: reachable area from evaluation. 
- Overlap of visible and reachable areas $r v$ :

$$
o_{r v}=\frac{M_{r v} \cap E_{r v}}{M_{r v} \cup E_{r v}}
$$

where $M_{r v}$ defines the reachable and visible area predicted by the model and $E_{r v}$ the area that was seen and reached during the evaluation run.

- Ratio of the visible area and $M_{r v}$ for the model:

$$
v_{M}=\frac{M_{r v}}{V}
$$

where $V$ defines the visible area seen through the microscope as defined in the previous section.

- Ratio of the visible area and $E_{r v}$ for the evaluation run:

$$
v_{E}=\frac{E_{r v}}{V}
$$

The first two indicators yield information about how well the model predicts the reachability. The last two ratios give more detail about the overall quality of the position of the eye. Fig. 10 shows the top view on the determined areas on the retina for the best position of the eye with no tilt of the robot for each considered rotation. It also illustrates the areas that are considered by the listed indicators. Table III shows the related quantitative results of the evaluation. Looking at the

\begin{tabular}{|l|l|l|l|l|}
\hline & $-10^{\circ}$ & $0^{\circ}$ & $30^{\circ}$ & avg. \\
\hline$o_{r}$ & 0.69 & 0.71 & 0.76 & 0.72 \\
\hline$o_{r v}$ & 0.84 & 0.86 & 0.92 & 0.87 \\
\hline$v_{M}$ & 0.76 & 0.87 & 0.69 & 0.77 \\
\hline$v_{E}$ & 0.63 & 0.74 & 0.63 & 0.67 \\
\hline
\end{tabular}

TABLE III

EVALUATION RESULTS WITHOUT TILTING THE ROBOT.

percentage of reachable areas within the visible area $v_{M}$ and $v_{E}$, the model yields better results than those obtained during the evaluation run. Clearly, this is because the RCM is set manually and used as an input for the model as well, not the maximum coverage is reached in the model. Nonetheless, the evaluation shows that the model is consistent and satisfactory results can be achieved.

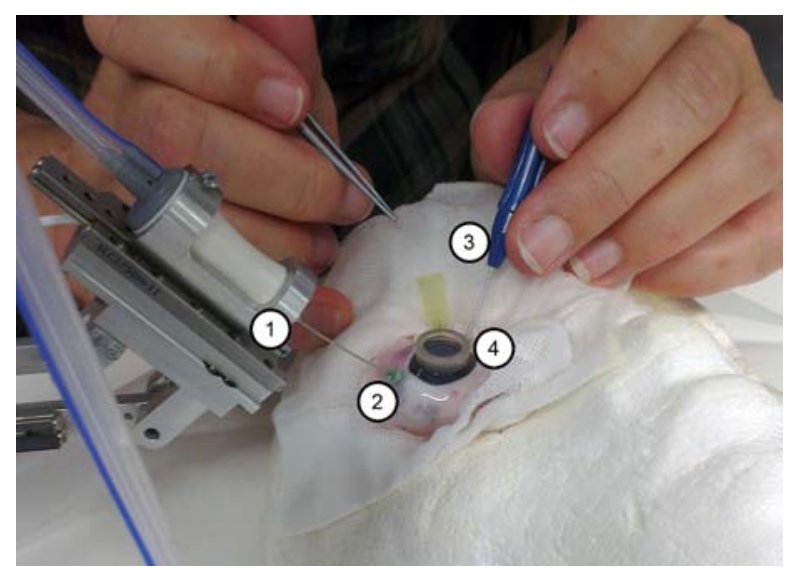

Fig. 11. The experimental setup: 1. tool gripper, 2. needle placed at the entry point on the surface of the eye, 3. tool for illumination, 4. additional lense for focusing on the retina with the microscope.
1) Automatic Location of the RCM: During an ophthalmic surgery the eye usually needs to be rotated to access different areas on the retina. This results in a relocation of the RCM on the surface of the eye. To achieve this, the surgeon normally follows a registration procedure. This can be done with the help of a marker on the instrument. The surgeon pulls the instrument out of the eye until the marker gets visible and then pushes a button on the $3 \mathrm{D}$ mouse. The $\mathrm{RCM}$ is then set to the position of the marker. However, this procedure is time consuming and distracts the surgeon from his actual task during an eye surgery. As a result, a method for automatically locating the RCM with the current setup is presented.

If the eye is assumed to be a simple sphere [17], it can be described with four points in Cartesian space. Before a procedure begins, the surgeon follows the previously explained registration procedure four times at different locations. That is, he rotates the eye to four different positions and pulls the instrument outside the eye until the marker gets visible. By pushing the button on the 3D mouse, the coordinates of the marker are recorded and after the fourth registration procedure the parameters of a sphere can be determined as explained in the following. The most general way of describing the geometry of a sphere is implicitly in the projective space $\mathbb{P}^{3}$ with the equation

$$
\mathbf{x}^{T} \mathbf{Q} \mathbf{x}=0
$$

with $\mathbf{x}$ representing a point in $\mathbb{P}^{3}$ and $\mathbf{Q}$ the quadric surface, which is given by the diagonal matrix $\mathbf{Q}=\operatorname{diag}(1,1,1,-1)$ (see e.g.[15]). A quadric transforms as

$$
\mathbf{Q}^{\prime}=\mathbf{T}^{T} \mathbf{Q} \mathbf{T}
$$

with

$$
\mathbf{T}=\left(\begin{array}{ll}
\mathbf{R} & \mathbf{t} \\
\mathbf{0} & 1
\end{array}\right)
$$

where $\mathbf{R}$ represents a rotation matrix and $\mathbf{t}$ a translational offset. In case of a sphere, $\mathbf{R}$ is a diagonal matrix i.e. $\operatorname{diag}(s, s, s)$ where $s$ is a scaling factor. Thus the scaling of the sphere and its offset with respect to the robot base are encoded in $\mathbf{T}$. Given the four registered points, one can define four equations with $\mathbf{x}_{i}^{T} \mathbf{Q} \mathbf{x}_{i}=0$ to determine $s$ and t. For setting the RCM automatically when it is moved, the intersection of the tool shaft with the sphere needs to be calculated. A point on the tool shaft can be expressed as

$$
\mathbf{p}_{l}=\mathbf{p}_{5}+\hat{\lambda} \mathbf{p}_{\text {tool }}, \hat{\lambda} \in \mathbb{R}
$$

To determine the point of intersection, one has to calculate the value of $\hat{\lambda}$ via the equation

$$
\left(\begin{array}{ll}
\mathbf{p}_{l}^{T} & 1
\end{array}\right) \mathbf{Q}^{\prime}\left(\begin{array}{c}
\mathbf{p}_{l} \\
1
\end{array}\right)=0
$$

This yields at most two results for $\hat{\lambda}$ if there is an intersection with the sphere. The desired solution for $\hat{\lambda}$ can then be determined from the orientation of the eye within the workspace. In our case, the eye points upwards along the $x$-axis and thus 


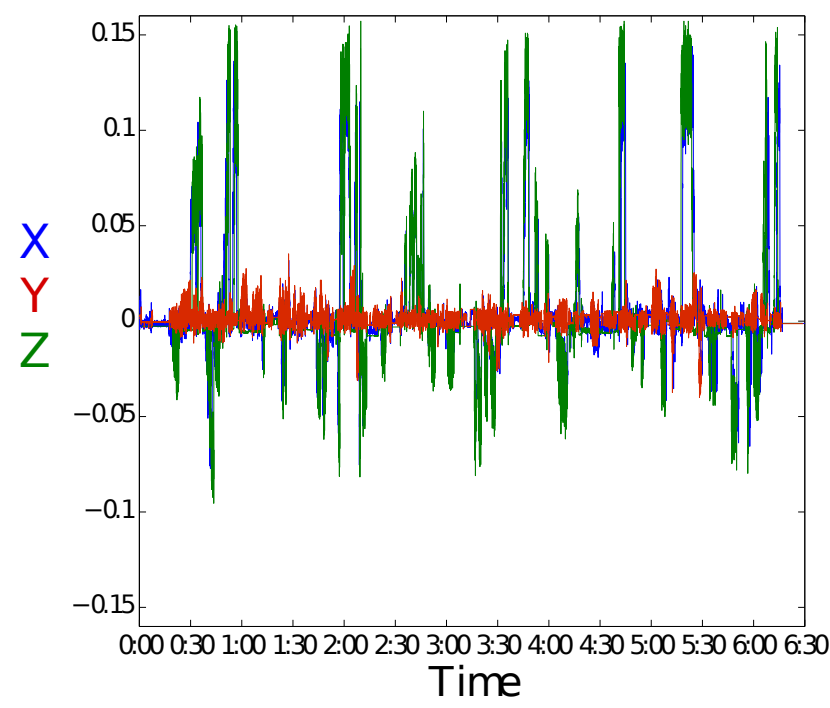

Fig. 12. The error of the RCM from raw position data of the linear actuators.

the smaller value of $\hat{\lambda}$ is the correct solution. As a result, the position of the RCM is defined as

$$
\mathbf{p}_{\text {rcm }}=\mathbf{p}_{5}+\hat{\lambda} \mathbf{p}_{\text {tool }}
$$

\section{A. Clinical Experiments}

Ex-vivo clinical experiments was performed at ophthalmic operation theater of klinikum rechts der isar, Munich (See Fig. 11). Using the standard $23 \mathrm{G}$ trocar system the cannula was docked into the porcine eye. The robot introduced the ophthalmic tool, which in this case was a micro forceps, through the cannula. The RCM point (incision point) was detected at the beginning of insertion phase and it was chosen by pressing a bottom on input device. There is also the ability of changing the RCM point by pressing the bottom, moving it to the new position (e.g. for eye rotation) and selecting of the new point by pressing the bottom once more. Fig. 12 shows the error from the raw position data in $x, y$ and $z$ direction, which was captured during clinical experiments. It is seen that only a maximum error of $0.16 \mathrm{~mm}$ is reached, which is sufficient to guarantee that there is no critical stress on the surface of the Sclera while intraocular manipulation is taking place.

\section{CONCLUSION}

The virtual fixture control of the hybrid parallel-serial micromanipulator has been investigated in this paper. The feasibility of this feature, the method of implementation, modeling and experimental validation are the elements of this investigation. It has also been shown that the distribution of visible-accessible intraocular points is highly dependent on the location of the incision point and the robot. This dependency and the optimum locations of the robot, with respect to the eye, have been also discussed in this work. The virtualfixture control methods was simulated and experimentally evaluated.

\section{REFERENCES}

[1] R. MacHemer: The development of pars plana vitrectomy, a personal account, In: Graefe's archive for clinical and experimental ophthalmology 233 (8) (1995)

[2] M. A. Nasseri et al.: The Introduction of a New Robot for Assisting in Ophthalmic Surgery, In: Proceeding of 35th Annual International Conference of the IEEE EMBC. pp. 5682-5685, (2013)

[3] T. Ueta et al.: Robot-assisted vitreoretinal surgery: Development of a prototype and feasibility studies in an animal model. Ophthalmology 116, 1538-1543 (2009)

[4] Meenink, T.: Vitreo-Retinal eye surgery robot: sustainable precision. Phd thesis, TU Eindhoven, ISBN:978-90-386-2800-4,(2011).

[5] A. Uneri et al.: New steady-hand eye robot with micro-force sensing for vitreoretinal surgery. In: Proceedings of the 2010 3rd IEEE RAS and EMBS International Conference on Biomedical Robotics and Biomechatronics. pp. 814-819 (2010)

[6] Nair, S. et al.: Embedded middleware and hard real-time architecture for robot assisted ophthalmic surgery. In: Proceedings of The Hamlyn Symposium on Medical Robotics, pp. 83-84. (2013)

[7] N. Aghakhani et al. :Task Control with Remote Center of Motion Constraint for Minimally Invasive Robotic Surgery. In: Proceedings of the IEEE Int. Conf. on Robotics and Automation (ICRA) (2013)

[8] Azimian, H. Patel, R.V. and Naish, M.D. :On constrained manipulation in robotics-assisted minimally invasive surgery. In: Proceedings of the IEEE Biomedical Robotics and Biomechatronics (BioRob), pp. 650$655(2010)$

[9] S. Rizzo, F. Patelli, and D. R. Chow. : Vitreo-retinal Surgery. SpringerVerlag Berlin Heidelberg, ISBN: 978-3-540-69461-8, progress iii edition (2009).

[10] Y. Nakamura et al. : Task-priority based redundancy control of robot manipulators In: The International Journal of Robotics Research-Sage Publications, vol. 6, pp. 3-15 (1987).

[11] Seraji, H. : Configuration control of redundant manipulators: theory and implementation In: Transactions on Robotics and Automation, IEEE, vol. 5/4, pp. 472-4903 (1989).

[12] Baillieul, J. : Kinematic programming alternatives for redundant manipulators In: Proceedings of IEEE International Conference on Robotics and Automation, vol. 2, pp. $722-728$ (1985).

[13] M. W. Charles and N. Brown : Dimensions of the human eye relevant to radiation protection (dosimetry) In: Journal of Physics in Medicine and Biology, vol. 20, pp. 202 (1975).

[14] Locke, R.C.O. and Patel, R.V. : Optimal Remote Center-of-Motion Location for Robotics-Assisted Minimally-Invasive Surgery In: Proceedings of IEEE International Conference on Robotics and Automation, pp. 1900-1905 (2007).

[15] Hartley, R. I. and Zisserman, A. : Multiple View Geometry in Computer Vision ,Cambridge University Press, ISBN: 0521540518 (2004).

[16] M. Ali Nasseri et al. : Clinical motion tracking and motion analysis during ophthalmic surgery using electromagnetic tracking system In: Proceedings of the 5th International Conference on BioMedical Engineering and Informatics (BMEI), pp 1006?1010 (2012).

[17] Sigal, I.A. and Flanagan, J.G. and Tertinegg, I. and Ethier, C.R. : Finite element modeling of optic nerve head biomechanics In: Investigative ophthalmology \& visual science, pp $4378 ? 4387$ (2004).

[18] Wei, W. Goldman, R. Simaan, N. Fine, H. Stanley, C. "Design and Theoretical Evaluation of Micro-Surgical Manipulators for Orbital Manipulation and Intraocular Dexterity," Robotics and Automation, 2007 IEEE International Conference on , vol., no., pp.3389,3395, 1014 April 2007

[19] H. Yu, Jin-Hui S. Joos, K.M. Simaan, N., "Design, calibration and preliminary testing of a robotic telemanipulator for OCT guided retinal surgery," Robotics and Automation (ICRA), 2013 IEEE International Conference on , vol., no., pp.225,231, 6-10 May 2013 